\title{
Impact vibration attenuation for a flexible robotic manipulator through transfer and dissipation of energy
}

\author{
Yushu Bian* and Zhihui Gao \\ School of Mechanical Engineering and Automation, Beihang University, Beijing, China
}

Received 4 August 2012

Revised 14 January 2013

Accepted 22 January 2013

\begin{abstract}
Due to the presence of system flexibility, impact can excite severe large amplitude vibration responses of the flexible robotic manipulator. This impact vibration exhibits characteristics of remarkable nonlinearity and strong energy. The main goal of this study is to put forward an energy-based control method to absorb and attenuate large amplitude impact vibration of the flexible robotic manipulator. The method takes advantage of internal resonance and is implemented through a vibration absorber based on the transfer and dissipation of energy. The addition of the vibration absorber to the flexible arm generates a coupling effect between vibration modes of the system. By means of analysis on 2:1 internal resonance, the exchange of energy is proven to be existent. The impact vibrational energy can be transferred from the arm to the absorber and dissipated through the damping of the absorber. The results of numerical simulations are promising and preliminarily verify that the method is feasible and can be used to combat large amplitude impact vibration of the flexible manipulator undergoing rigid motion.
\end{abstract}

Keywords: Flexible robotic manipulator, vibration, impact, internal resonance

\section{Introduction}

With the development of robotic manipulators toward light weight, heavy load, high speed and large work space, dynamic effects resulted from their structural flexibility cannot be neglected. How to effectively overcome these adverse influences on work performance is an important problem.

In the area of motion control for flexible robotic manipulators, different ways have been sought and important advancements have been achieved [1-3]. On the other hand, since most of robotic manipulators are required to make physical contact as part of their jobs, such as assembly, repair and capture, impact phenomenon always arises inevitably. Also, various disturbances from the environment, like unexpected collision from other objects, result in impact. Generally, characteristics of impact are very brief duration, high force levels, rapid dissipation of energy and large accelerations/decelerations [4]. As far as the flexible manipulator is concerned, impact can cause abrupt change in system velocities, thereby exciting severe post-impact vibration responses. Furthermore, since the manipulator is required to keep moving after the collision, new vibration responses inevitably arise and are accumulated on the existing impact vibration responses. Therefore, vibration control of the flexible manipulator undergoing an impact collision is both theoretically challenging and of practical importance.

\footnotetext{
*Corresponding author: Yushu Bian, School of Mechanical Engineering and Automation, Beihang University, Beijing, China. E-mail: bian_bys@buaa.edu.cn.
} 
In past decades, impact modeling for the flexible manipulator has received considerable attention [4-7]. However, in contrast to a number of papers on impact control for the rigid manipulator [8-13], insufficient research appears to have been conducted on impact vibration reduction for the flexible manipulator [14-18]. Most of control algorithms rely on accurate contact position and collision time, but in some real working environments like outer space, collision from micrometeoroids are usually unpredictable. In addition, due to system flexibility, the duration between the time in which a collision occurs and that in which the system detects the collision, known as the collision detection time, is long, thereby resulting in the delay of reaction strategy. Therefore, control methods based on the collision detection will face challenges. More importantly, impact energy always excites large amplitude vibration. This large amplitude may enhance the effects of the nonlinearities in the system. In this case, many nonlinear terms that could have been ignored in some control methods must be taken into account again. Otherwise, irrational linearization may result in not only calculation inaccuracy but also fundamental mistakes.

Although a large number of active control schemes can be used to control vibration of the flexible manipulator [19, 20], most of them, especially those based on smart materials like piezoelectric ceramic and shape memory alloy etc., probably face challenges when dealing with impact vibration problem. In the post-impact phase, huge impact energy may excite severe large amplitude vibration of the flexible manipulator. As we know, active control methods mainly depend on external energy to suppress vibration. In this way, they have to afford much more energy to combat with this large amplitude vibration. Obviously, it is not always advisable. Furthermore, since output powers of many smart material actuators are limited, it is difficult for them to provide sufficient energy to resist this large amplitude vibration. Therefore, compared with active suppression methods, vibration absorption methods based on the transfer and dissipation of vibrational energy seem more feasible to overcome large amplitude impact vibration.

Since large amplitude impact vibration of the flexible manipulator exhibits characteristics of remarkable nonlinearity and strong energy, it is necessary to seek an effective vibration absorption method based on appropriate nonlinear principle. For a multi-degree-of-freedom nonlinear vibrating system, internal resonance is one of typical nonlinear characteristics of modal interaction. For example, if two natural frequencies of the linear portion of a set of nonlinear equations of motion are commensurable or nearly commensurable (i.e. $n_{1} \omega_{1}=n_{2} \omega_{2}$, where $n_{1}$ and $n_{2}$ are integers, $\omega_{1}$ and $\omega_{2}$ are natural frequencies), internal resonance will occur [21]. At the state of internal resonance, nonlinear coupling will provide a bridge to facilitate a transfer of vibrational energy between the related modes. Although there are a lot of papers concerning internal resonance phenomenon [22-27], most of them strive to avoid resonance conditions whereas seldom capitalize on internal resonance for control applications.

In fact, studying internal resonance from a different perspective can help find new ways for vibration control. Golnaraghi $[28,29]$ was one of the first researchers to employ internal resonance to control vibration of a flexible cantilever beam. Based on internal resonance, the beam vibration was reduced by removing its vibrational energy and dissipating it through the slider motion actuated by a motor with position and velocity feedback. Tuer et al. [30] and Duquette et al. [31] conducted theoretical and experimental studies, and regulated the motion of a similar beam by means of a rotational internal resonance controller at the tip of the beam. In addition, Tuer et al. [32] proposed two control strategies to regulate a single-degree-of-freedom second order system based on internal resonance. Furthermore, Oueini et al. [33] conducted numerical simulations in regulating vibration of a two-degree-of- freedom structure. Afterwards, Oueini et al. [34] finished an experimental study in which the controller was implemented by analog electronic components. From these studies, it is shown that internal resonance seems to be a promising tool for vibration control of the flexible structure without rigid motion.

In aforementioned studies, in order to create a simple single-degree-of-freedom model for analysis, the controlled cantilever beam, which is inherently flexible, is assumed as a rigid beam attached by a linear torsional spring. Obviously, this lumped flexibility model is not suitable for the analysis of a robotic manipulator with long and thin flexible links; instead, a distributed flexibility model is advisable. Besides, these studies mainly focused on the flexible structure but did not examine the flexible mechanism. In fact, one of important differences between them is that the former has no large-scale nominal motion (i.e. rigid motion) but the latter has. As a result, compared with the flexible structure, the flexible mechanism usually exhibits much more complex dynamic behaviors due to coupling effects between its rigid motion and flexural motion. In addition, the controlled cantilever beam is simplified as a linear vibration model and a supplementary system (i.e. vibration controller) is added to artificially generate nonlinear coupling. However, since the flexible arm is a nonlinear vibrating system in nature, its dynamic coupling with a supplementary system will become more complicated. More importantly, although the above methods used 
internal resonance to transfer vibrational energy from the flexible cantilever beam to the supplementary system, the exchanged vibrational energy was suppressed by the actuators of vibration controllers (e.g. motors). This means that these methods still relied on external energy suppressing vibration response. Therefore, in essence they belong to active control methods.

To our knowledge, internal resonance seems not to have been utilized in dynamic control of the flexible manipulator so far. Whether internal resonance can be successfully established for the flexible manipulator undergoing rigid motion is not verified. Especially, its theoretical feasibility of combating with large amplitude impact vibration of the flexible manipulator is not explored. The main goal of this study is to put forward an energy-based control method to absorb and attenuate large amplitude impact vibration of the flexible robotic manipulator. The method takes advantage of internal resonance and is implemented through a vibration absorber based on the transfer and dissipation of energy. The addition of a vibration absorber to the flexible arm generates a coupling effect between vibration modes of the system. By means of analysis on 2:1 internal resonance, the exchange of energy is proven to be existent. The impact vibrational energy can be transferred from the arm to the absorber and dissipated through the damping of the absorber. Since the method aims to regulate internal energy transfer of the flexible manipulator, it does not necessarily rely on accurate contact position and collision time.

\section{Impact dynamics}

Based on Kane's method and the assumed-modes method, dynamics equations of the flexible manipulator are derived and can be written as follows [35]:

$$
M_{s} \ddot{\chi}+C_{s} \dot{\chi}+K_{s} \chi=Q
$$

where $\boldsymbol{M}_{s} \in \boldsymbol{R}^{n \times n}$ is the system mass matrix; $\boldsymbol{C}_{s} \in \boldsymbol{R}^{n \times n}$ is the system damping matrix; $\boldsymbol{K}_{s} \in \boldsymbol{R}^{n \times n}$ is the system stiffness matrix; $\boldsymbol{Q} \in \boldsymbol{R}^{n}$ is the sum of coriolis, gravitational, centripetal, and control torques; $\chi \in \boldsymbol{R}^{n}$ is the set of rigid and flexural degrees of freedom, $\chi^{T}=\left[\begin{array}{ll}\boldsymbol{q}^{T} & \boldsymbol{\varphi}^{T}\end{array}\right]^{T} ; \boldsymbol{q} \in \boldsymbol{R}^{n_{R}}$ is the vector describing the rigid joint angles; $\varphi \in R^{n_{F}}$ is the set of flexural displacement of links; $n$ is the total number of degrees of freedom, $n=n_{R}+n_{F} ; n_{R}$ is the number of joints; $n_{F}$ is the number of flexural degrees of freedom.

Equation (1) can be separated into two equations describing the dynamics of $\boldsymbol{q}$ and $\varphi$

$$
\begin{aligned}
& \boldsymbol{D} \ddot{\boldsymbol{q}}+\boldsymbol{U} \ddot{\boldsymbol{\varphi}}=\tau_{1}+\tau_{2} \\
& \boldsymbol{G} \ddot{\boldsymbol{q}}+\boldsymbol{M} \ddot{\varphi}+C \dot{\varphi}+\boldsymbol{K} \boldsymbol{\varphi}=\boldsymbol{f}
\end{aligned}
$$

where $\boldsymbol{D} \in \boldsymbol{R}^{n_{R} \times n_{R}}, \boldsymbol{U} \in \boldsymbol{R}^{n_{R} \times n_{F}}, \boldsymbol{G} \in \boldsymbol{R}^{n_{F} \times n_{R}}, \boldsymbol{M} \in \boldsymbol{R}^{n_{F} \times n_{F}}$ are block matrices that form $\boldsymbol{M}_{s} ; \boldsymbol{\tau}_{1} \in \boldsymbol{R}^{n_{R}}$ is the set of control torques applied to the joints; $\tau_{2} \in \boldsymbol{R}^{n_{R}}$ is the rigid component of the nonlinear torque; $C \in$ $\boldsymbol{R}^{n_{F} \times n_{F}} ; \boldsymbol{K} \in \boldsymbol{R}^{n_{F} \times n_{F}} ; \boldsymbol{f} \in \boldsymbol{R}^{n_{F}}$ is the flexural component of the nonlinear torque.

The real impact process is quite complex in fact. Since our research is focused on reduction of large amplitude impact vibration, it is assumed here that: (a) impact time is infinitesimal; (b) impact is modeled as the point contact; (c) impact process is simplified as an impulse force; (d) the colliding object is treated as a point mass.

Supposing a moving object collides with the flexible arm at a certain point $P$, the impact force resulted from this collision is $\boldsymbol{f}_{\Delta} \in \boldsymbol{R}^{3}$. The dynamics equations of the flexible manipulator undergoing impact can be written as:

$$
\begin{aligned}
& D \ddot{\boldsymbol{q}}+\boldsymbol{U} \ddot{\boldsymbol{\varphi}}=\tau_{1}+\tau_{2}+\boldsymbol{f}_{\Delta}^{I} \\
& \boldsymbol{G} \ddot{\boldsymbol{q}}+\boldsymbol{M} \ddot{\boldsymbol{\varphi}}+\boldsymbol{C} \dot{\boldsymbol{\varphi}}+\boldsymbol{K} \boldsymbol{\varphi}=\boldsymbol{f}+\boldsymbol{f}_{\Delta}^{I I}
\end{aligned}
$$

where

$$
\begin{aligned}
\boldsymbol{f}_{\Delta}^{I} & =\left\{\begin{array}{llll}
f_{\Delta 1}^{I} & f_{\Delta 2}^{I} & \ldots & f_{\Delta n_{R}}^{I}
\end{array}\right\}^{T} \\
\boldsymbol{f}_{\Delta}^{I I} & =\left\{\begin{array}{llll}
f_{\Delta 1}^{I I} & f_{\Delta 2}^{I I} & \ldots & f_{\Delta n_{F}}^{I I}
\end{array}\right\}^{T}
\end{aligned}
$$

$\boldsymbol{f}_{\Delta}^{I} \in \boldsymbol{R}^{n_{R}}$ and $\boldsymbol{f}_{\Delta}^{I I} \in \boldsymbol{R}^{n_{F}}$ are generalized active forces caused by $\boldsymbol{f}_{\Delta}$ and can be obtained using Kane's method, i.e. 


$$
\begin{aligned}
f_{\Delta j}^{I}=\boldsymbol{v}_{p j} \boldsymbol{f}_{\Delta} & \left(1 \leqslant j \leqslant n_{R}\right) \\
f_{\Delta\left(j-n_{R}\right)}^{I I} & =\boldsymbol{v}_{p j} \boldsymbol{f}_{\Delta} \quad\left(n_{R}+1 \leqslant j \leqslant n\right)
\end{aligned}
$$

where $\boldsymbol{v}_{p j} \in \boldsymbol{R}^{3}$ is the $j$-th partial speed of $\boldsymbol{v}_{p}, \boldsymbol{v}_{p}$ is the velocity of point $P$.

Since impact occurs in an infinitesimal period of time, if taking the integral of the dynamics Eqs (4) and (5) from $t$ to $t+\Delta t$, we obtain

$$
\begin{aligned}
& \int_{t}^{t+\Delta t} \boldsymbol{D} \ddot{\boldsymbol{q}} d t+\int_{t}^{t+\Delta t} \boldsymbol{U} \ddot{\boldsymbol{\varphi}} d t=\int_{t}^{t+\Delta t} \boldsymbol{\tau}_{1} d t+\int_{t}^{t+\Delta t} \boldsymbol{\tau}_{2} d t+\int_{t}^{t+\Delta t} \boldsymbol{f}_{\Delta}^{I} d t \\
& \int_{t}^{t+\Delta t} \boldsymbol{G} \ddot{\boldsymbol{q}} d t+\int_{t}^{t+\Delta t} \boldsymbol{M} \ddot{\boldsymbol{\varphi}} d t+\int_{t}^{t+\Delta t} \boldsymbol{C} \dot{\boldsymbol{\varphi}} d t+\int_{t}^{t+\Delta t} \boldsymbol{K} \boldsymbol{\varphi} d t=\int_{t}^{t+\Delta t} \boldsymbol{f} d t+\int_{t}^{t+\Delta t} \boldsymbol{f}_{\Delta}^{I I} d t
\end{aligned}
$$

Because all linear and angular velocities remain finite in this infinitesimally small period of the impact time, and there are no changes in positions or orientations of any bodies in the system as $\Delta t \rightarrow 0$, only the integrals involving $\ddot{\boldsymbol{q}}, \ddot{\boldsymbol{\varphi}}, \boldsymbol{f}_{\Delta}^{I}$ and $\boldsymbol{f}_{\Delta}^{I I}$ are not zero. From Eqs (10) and (11), we have

$$
\begin{aligned}
& \boldsymbol{D} \Delta \dot{\boldsymbol{q}}+\boldsymbol{U} \Delta \dot{\boldsymbol{\varphi}}=\int_{t}^{t+\Delta t} \boldsymbol{f}_{\Delta}^{I} d t \\
& \boldsymbol{G} \Delta \dot{\boldsymbol{q}}+\boldsymbol{M} \Delta \dot{\boldsymbol{\varphi}}=\int_{t}^{t+\Delta t} \boldsymbol{f}_{\Delta}^{I I} d t
\end{aligned}
$$

where $\Delta \dot{\boldsymbol{q}}=\dot{q}(t+\Delta t)-\dot{q}(t), \Delta \dot{\varphi}=\dot{\varphi}(t+\Delta t)-\dot{\varphi}(t)$.

According to the Momentum Theorem, we obtain

$$
\begin{array}{ll}
\int_{t}^{t+\Delta t} f_{\Delta j}^{I} d t=\int_{t}^{t+\Delta t} \boldsymbol{v}_{p j} \boldsymbol{f}_{\Delta} d t=\boldsymbol{v}_{p j} M_{0} \boldsymbol{v}_{0} & \left(1 \leqslant j \leqslant n_{R}\right) \\
\int_{t}^{t+\Delta t} f_{\Delta\left(j-n_{R}\right)}^{I I} d t=\int_{t}^{t+\Delta t} \boldsymbol{v}_{p j} \boldsymbol{f}_{\Delta} d t=\boldsymbol{v}_{p j} M_{0} \boldsymbol{v}_{0} & \left(n_{R}+1 \leqslant j \leqslant n\right)
\end{array}
$$

where $M_{0}$ is the mass of the moving object, $\boldsymbol{v}_{0}$ is relative velocity of the moving object with respect to the contact point of the arm.

According to Eqs (6), (7), (14) and (15), Eqs (12) and (13) can be written as

$$
\begin{aligned}
& D \Delta \dot{\boldsymbol{q}}+\boldsymbol{U} \Delta \dot{\varphi}=\boldsymbol{h}_{I} \\
& \boldsymbol{G} \Delta \dot{\boldsymbol{q}}+\boldsymbol{M} \Delta \dot{\varphi}=\boldsymbol{h}_{I I}
\end{aligned}
$$

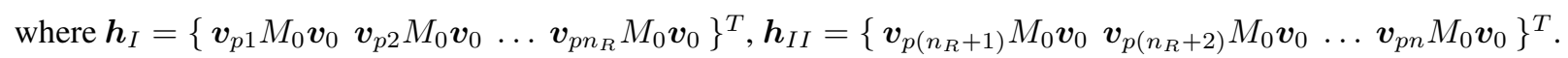
Incorporating Eqs (16) and (17), we obtain

$$
M_{s} \Delta \dot{\chi}=h
$$

where

$$
\boldsymbol{M}_{s}=\left[\begin{array}{cc}
\boldsymbol{D} & \boldsymbol{U} \\
\boldsymbol{G} & \boldsymbol{M}
\end{array}\right], \quad \Delta \dot{\boldsymbol{\chi}}=\left[\begin{array}{cc}
\Delta \dot{\boldsymbol{q}}^{T} & \Delta \dot{\boldsymbol{\varphi}}^{T}
\end{array}\right]^{T}, \quad \boldsymbol{h}=\left[\begin{array}{ll}
\boldsymbol{h}_{I}^{T} & \boldsymbol{h}_{I I}^{T}
\end{array}\right]^{T} .
$$

Equation (18) is the impact dynamics equation of the flexible manipulator, describing the relationship between the instantaneous velocity increment and the impulsive contact force. The final values $\Delta \dot{\psi}$ of Eq. (18) are used as the initial conditions of the post-impact phase. 


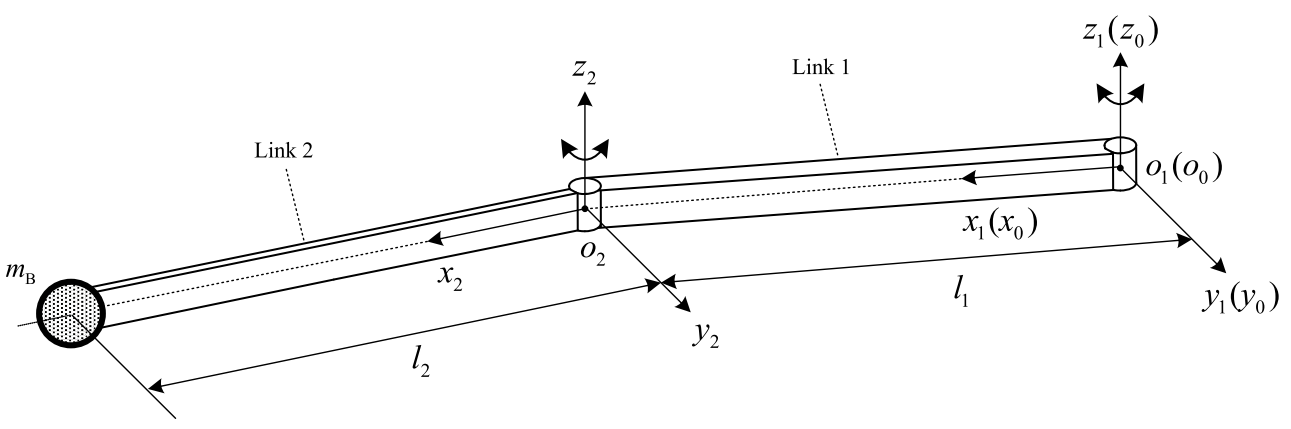

Fig. 1. Model of the flexible manipulator.

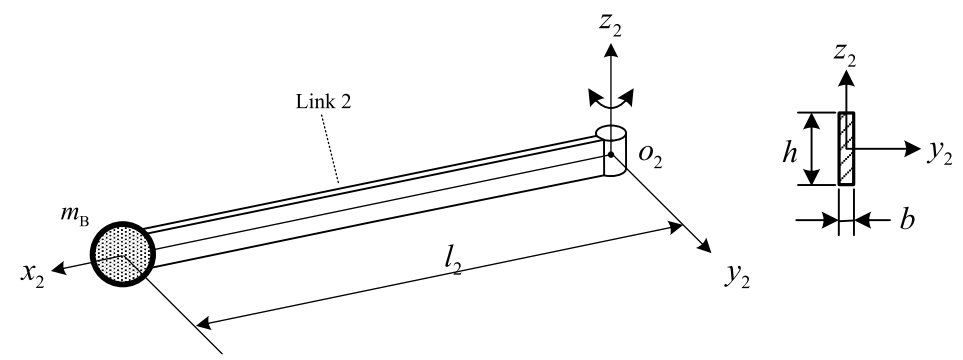

(a)

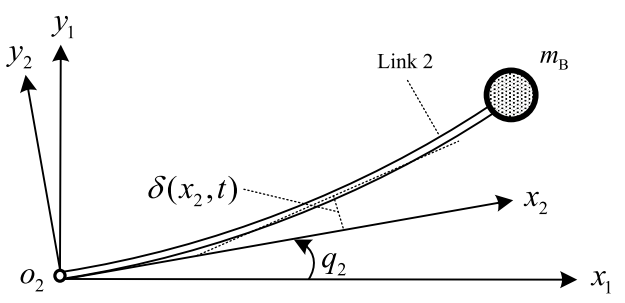

(b)

Fig. 2. Model of the flexible link.

\section{Mathematical model of the flexible manipulator with vibration absorber}

In this study, a simplified two-link robotic arm model with last link flexible is employed to examine the effectiveness of the proposed technique, as shown in Fig. 1. In the preliminary stage, this simplification can help decrease computational complexity while retain basic features of the flexible manipulator. Link1 is a uniform rigid beam with the length $l_{1}$, the square cross-section of side length $s$. Link2 is a uniform Euler-Bernoulli flexible beam with the length $l_{2}$, the rectangle cross-section of height $h$ and width $b$, a tip mass $m_{B}$, as shown in Fig. 2. Only the in-plane flexural deformation $\delta\left(x_{2}, t\right)$ about $y_{2}$ axis in the plane $o_{2} x_{2} y_{2}$ is considered, where $t$ is time. The joint $o_{1}$ and $o_{2}$ is controlled to provide large-scale rigid motion (i.e. nominal motion) of the arm around the $z_{1}$ and $z_{2}$ axis, denoted by $q_{1}$ and $q_{2}$ respectively.

To reduce vibration of the flexible arm, another vibrating system, used as a vibration absorber, is attached to the flexible arm at $x_{2}=r$, as shown in Fig. 3. The absorber consists of a rigid link, a flexible joint and a damper. The uniform rigid link with mass $m_{3}$ and centroid position $r_{c 3}$, is perpendicularly connected, through the flexible joint and the damper, to the link2 in the plane $\mathrm{o}_{2} x_{2} y_{2}$. It should be noted that the rigid link is not unique form and other configurations with certain rotary inertia are acceptable in the absorber. The flexibility and damping of the absorber are modeled using a torsional spring/damper configuration. When the flexible link deforms in the plane $o_{2} x_{2} y_{2}$, the angle of the tangent of the flexible link at $x_{2}=r$ with respect to $x_{2}$ axis is denoted by $\alpha$, as shown in Fig. 3(b), i.e.

$$
\left.\alpha \approx \frac{\partial \delta\left(x_{2}, t\right)}{\partial x_{2}}\right|_{x_{2}=r}
$$

When the flexible joint deforms paralleling the plane $o_{2} z_{2} y_{2}$, the angle of the rigid link with respect to $y_{2}$ axis is denoted by $\beta$, as shown in Fig. 3(a). Obviously, the vibration absorber increases the structural degrees of freedom by one, i.e. $\beta$. 


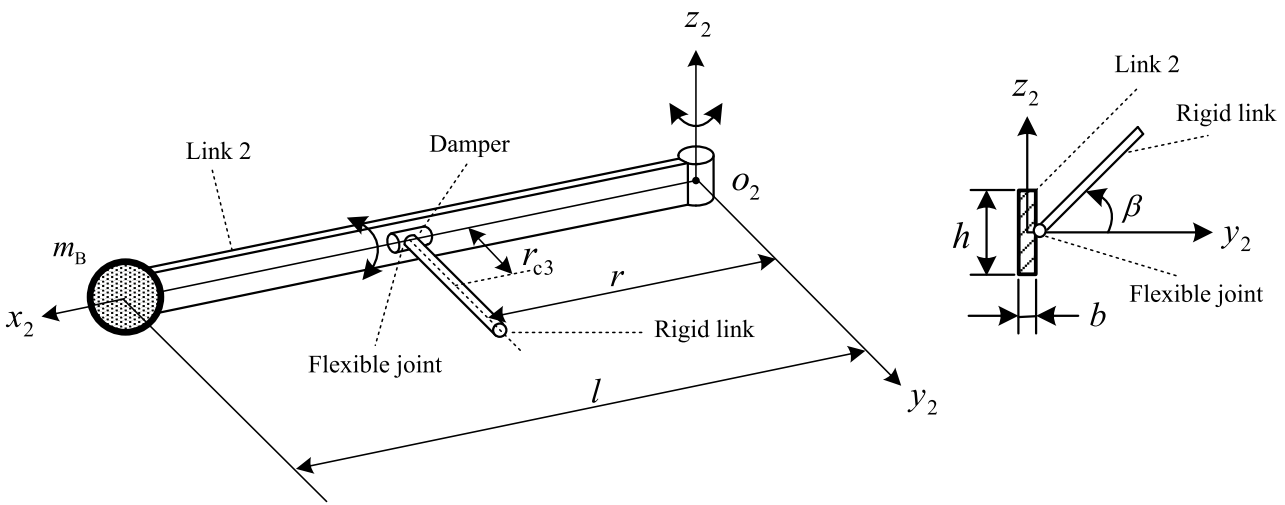

(a)

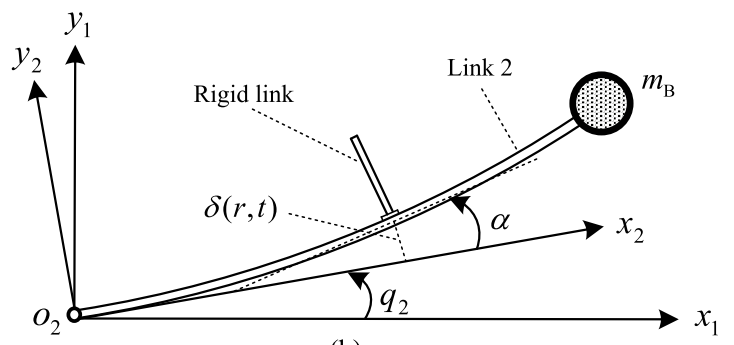

(b)

Fig. 3. Model of the flexible link with a vibration absorber.

\section{Equations of motion}

According to the assumed-modes theory, transverse deformation of the link 2 in the plane $o_{2} x_{2} y_{2}$ is expressed in terms of the mode shapes

$$
\delta\left(x_{2}, t\right)=\sum_{i=1}^{n_{F}} u_{i}\left(x_{2}\right) \varphi_{i}(t)
$$

where $\varphi_{i}(t)$ is the $i$-th modal coordinate describing deformation of the flexible link, $u_{i}\left(x_{2}\right)$ is the $i$-th mode shape satisfying certain geometric and force boundary conditions.

In this study, only the fundamental mode of the link is considered due to its most contribution to the vibration response in common cases. In this case, substituting Eq. (20) into Eq. (19) yields

$$
\alpha \approx \tilde{\beta}_{r} \varphi_{1}(t)
$$

where

$$
\tilde{\beta}_{r}=\left.\frac{d u_{1}\left(x_{2}\right)}{d x_{2}}\right|_{x_{2}=r}
$$

In addition, the axial displacement resulted from the transverse bending of the link is taken into account, i.e.

$$
v\left(x_{2}\right)=-\frac{1}{2} \int_{0}^{x_{2}}\left(\frac{\partial \delta(\vartheta, t)}{\partial \vartheta}\right)^{2} d \vartheta=-\frac{1}{2} B_{1} \varphi_{1}^{2}
$$

where $\vartheta$ is a dummy variable and

$$
B_{1}=\int_{0}^{x_{2}}\left(\frac{d u_{1}(\vartheta)}{d \vartheta}\right)^{2} d \vartheta
$$


In order to damp out vibration response of the fundamental mode of the link using the absorber, the coupling effect between the fundamental mode coordinate $\varphi_{1}$ of the link and the flexural degree of freedom $\beta$ of the absorber is mainly concerned. Therefore, based on Kane's method and the assumed-modes theory, the flexible dynamics equations concerning $\varphi_{1}$ and $\beta$ are derived in the case of zero gravity and can be written as

$$
\begin{aligned}
m_{11} \ddot{\varphi}_{1}+c_{11} \dot{\varphi}_{1}+\left(k_{11}-b_{1} \ddot{q}_{1}-b_{2} \ddot{q}_{2}\right) \varphi_{1}= & b_{3} \dot{\varphi}_{1}^{2}+b_{4} \varphi_{1} \ddot{\varphi}_{1}+b_{5} \dot{\beta}^{2}+b_{5} \beta \ddot{\beta}-b_{6} \ddot{q}_{1}-b_{7} \ddot{q}_{2}+b_{8}\left(\dot{q}_{1}\right. \\
& \left.+\dot{q}_{2}\right)^{2}-b_{9} \dot{q}_{1}^{2}+f_{1}\left(\varphi_{1}, \dot{\varphi}_{1}, \ddot{\varphi}_{1}, \beta, \dot{\beta}, \ddot{\beta}, \dot{q}_{1}, \ddot{q}_{1}, \dot{q}_{2}, \ddot{q}_{2}\right) \\
m_{22} \ddot{\beta}+c_{22} \dot{\beta}+\left(k_{22}-b_{10} \ddot{q}_{1}-b_{11} \ddot{q}_{2}\right) \beta= & b_{5} \beta \ddot{\varphi}_{1}+f_{2}\left(\varphi_{1}, \dot{\varphi}_{1}, \ddot{\varphi}_{1}, \beta, \dot{\beta}, \ddot{\beta}, \dot{q}_{1}, \ddot{q}_{1}, \dot{q}_{2}, \ddot{q}_{2}\right)
\end{aligned}
$$

where $m_{11}=B_{2}+m_{3}\left(u_{1 r}^{2}+\tilde{\beta}_{r}^{2} r_{c 3}^{2}\right)+m_{B} u_{1 l_{2}}^{2} ; B_{2}=\rho_{2} \int_{0}^{l_{2}} u_{1}^{2}\left(x_{2}\right) d x_{2} ; u_{1 r}=\left.u_{1}\left(x_{2}\right)\right|_{x_{2}=r} ; u_{1 l_{2}}=$ $\left.u_{1}\left(x_{2}\right)\right|_{x_{2}=l_{2}} ; c_{11}$ is the damping of the link2; $k_{11}=E_{2} I_{2} \int_{0}^{l_{2}} \frac{d^{4} u_{1}\left(x_{2}\right)}{d x_{2}^{4}} u_{1}\left(x_{2}\right) d x_{2} ; b_{1}=m_{3} r_{c 3}\left(\tilde{\beta}_{r}^{2} r-B_{1 r}\right)+$ $m_{3} l_{1}\left(\tilde{\beta}_{r}^{2} r_{c 3} \cos q_{2}+B_{1 r} \sin q_{2}\right) ; B_{1 r}=\left.B_{1}\left(x_{2}\right)\right|_{x_{2}=r}$;

$$
\begin{aligned}
& b_{2}=m_{3} r_{c 3}\left(\tilde{\beta}_{r}^{2} r-B_{1 r}\right) ; b_{3}=m_{3} \tilde{\beta}_{r} r_{c 3}\left(u_{1 r} \tilde{\beta}_{r}-B_{1 r}\right) ; b_{4}=2 m_{3} \tilde{\beta}_{r} r_{c 3}\left(u_{1 r} \tilde{\beta}_{r}-B_{1 r}\right) ; b_{5}=m_{3} u_{1 r} r_{c 3} ; \\
& b_{6}=m_{3}\left(u_{1 r} r+\tilde{\beta}_{r} r_{c 3}^{2}\right)-m_{3} l_{1}\left(\tilde{\beta}_{r} r_{c 3} \sin q_{2}-u_{1 r} \cos q_{2}\right)+l_{1} B_{4} \cos q_{2}+B_{3}+m_{B} u_{1 l_{2}}\left(l_{1} \cos q_{2}+l_{2}\right) ; \\
& B_{3}=\rho_{2} \int_{0}^{l_{2}} u_{1}\left(x_{2}\right) x_{2} d x_{2} ; b_{7}=m_{3}\left(u_{1 r} r+\tilde{\beta}_{r} r_{c 3}^{2}\right)+B_{3}+m_{B} u_{1 l_{2}} l_{2} ; b_{8}=m_{3} r_{c 3}\left(u_{1 r}-\tilde{\beta}_{r} r\right) ; \\
& b_{9}=m_{3} l_{1}\left(u_{1 r} \sin q_{2}+\tilde{\beta}_{r} r_{c 3} \cos q_{2}\right)+l_{1} B_{4} \sin q_{2} ; B_{4}=\rho_{2} \int_{0}^{l_{2}} u_{1}\left(x_{2}\right) d x_{2} ; m_{22}=I_{z z}^{3}+m_{3} r_{c 3}^{2} ; k_{22}=k ;
\end{aligned}
$$

$b_{10}=m_{3} r_{c 3}\left(r+l_{1} \cos q_{2}\right) ; b_{11}=m_{3} r_{c 3} r ; k$ is the torsional stiffness of the flexible joint; $c_{22}$ is the damping of the vibration absorber; $\rho_{2}$ is mass per length of the link2; $E_{2}$ is the Young's modulus of the link2; $E_{2} I_{2}$ is flexural rigidity of the link2; $I_{z z}^{3}$ is the moment of inertial of the rigid link with respect to its centroid axis.

In the following section, modal interaction effect between the fundamental mode of the flexible link and the vibration mode of the absorber will be investigated.

\section{Nondimensional and scaled equations of motion}

The first step in the analysis is to nondimensionalize the equations of motion. By means of the transformations

$$
\varphi^{*}=\frac{\varphi_{1}}{l_{2}}, \quad \beta^{*}=\beta, \quad t^{*}=\omega_{\beta} t, \quad q_{1}^{*}=q_{1}, \quad q_{2}^{*}=q_{2}
$$

where $\omega_{\beta}=\sqrt{k_{22} / m_{22}}$;

the equations of motion Eqs (25) and (26) are nondimensionalized to yield

$$
\begin{aligned}
\ddot{\varphi}^{*}+\eta_{11} \dot{\varphi}^{*}+\left(\omega_{\varphi \beta}^{2}-d_{1} \ddot{q}_{1}^{*}-d_{2} \ddot{q}_{2}^{*}\right) \varphi^{*}= & d_{3} \dot{\varphi}^{* 2}+d_{4} \ddot{\varphi}^{*} \varphi^{*}+d_{5} \dot{\beta}^{* 2}+d_{5} \ddot{\beta}^{*} \beta^{*}-d_{6} \ddot{q}_{1}^{*}-d_{7} \ddot{q}_{2}^{*}+d_{8}\left(\dot{q}_{1}^{*}\right. \\
& \left.+\dot{q}_{2}^{*}\right)^{2}-d_{9} \dot{q}_{1}^{* 2}+f_{3}\left(\varphi^{*}, \dot{\varphi}^{*}, \ddot{\varphi}^{*}, \beta^{*}, \dot{\beta}^{*}, \ddot{\beta}^{*}, \dot{q}_{1}^{*}, \ddot{q}_{1}^{*}, \dot{q}_{2}^{*}, \ddot{q}_{2}^{*}\right) \\
\ddot{\beta}^{*}+\eta_{22} \dot{\beta}^{*}+\left(1-d_{10} \ddot{q}_{1}^{*}-d_{11} \ddot{q}_{2}^{*}\right) \beta^{*}= & d_{12} \beta^{*} \ddot{\varphi}^{*}+f_{4}\left(\varphi^{*}, \dot{\varphi}^{*}, \ddot{\varphi}^{*}, \beta^{*}, \dot{\beta}^{*}, \ddot{\beta}^{*}, \dot{q}_{1}^{*}, \ddot{q}_{1}^{*}, \dot{q}_{2}^{*}, \ddot{q}_{2}^{*}\right)
\end{aligned}
$$

where

$$
\begin{aligned}
& \eta_{11}=c_{11} /\left(m_{11} \omega_{\beta}\right) ; \omega_{\varphi \beta}=\omega_{\varphi} / \omega_{\beta} ; \omega_{\varphi}^{2}=k_{11} / m_{11} ; d_{1}=b_{1} / m_{11} ; d_{2}=b_{2} / m_{11} ; d_{3}=b_{3} l_{2} / m_{11} ; \\
& d_{4}=l_{2} b_{4} / m_{11} ; \\
& d_{5}=b_{5} /\left(m_{11} l_{2}\right) ; d_{6}=b_{6} /\left(m_{11} l_{2}\right) ; d_{7}=b_{7} /\left(m_{11} l_{2}\right) ; d_{8}=b_{8} /\left(m_{11} l_{2}\right) ; d_{9}=b_{9} /\left(m_{11} l_{2}\right) \\
& \eta_{22}=c_{22} /\left(m_{22} \omega_{\beta}\right) ;
\end{aligned}
$$

$d_{10}=b_{10} / m_{22} ; d_{11}=b_{11} / m_{22} ; d_{12}=l_{2} b_{5} / m_{22} ;(\cdot)$ represents derivatives with respect to the nondimensional time $t^{*}$. 
In order to determine the effect of the nonlinearities of Eqs (28) and (29), it is necessary to scale the equations. A scaling factor $\varepsilon$, where $\varepsilon \ll 1$, is introduced to represent the order of nonlinear terms and coupling. It is chosen so that the nonlinearities are at least one order of magnitude smaller than the linear undamped portions. Therefore, the following transformations are used

$$
\varphi^{*}=\varepsilon \phi, \quad \beta^{*}=\varepsilon \psi, \quad \dot{q}_{1}^{*}=\varepsilon \dot{\Omega}_{1}, \dot{q}_{2}^{*}=\varepsilon \dot{\Omega}_{2}
$$

which implies that $\phi, \psi, \dot{\Omega}_{1}$ and $\dot{\Omega}_{2}$ are of the same order of magnitude.

Then substituting Eq. (30) into Eqs (28) and (29), yields

$$
\begin{aligned}
\varepsilon \ddot{\phi}+\varepsilon \eta_{11} \dot{\phi}+\varepsilon\left(\omega_{\varphi \beta}^{2}-\varepsilon d_{1} \ddot{\Omega}_{1}-\varepsilon d_{2} \ddot{\Omega}_{2}\right) \phi= & \varepsilon^{2} d_{3} \dot{\phi}^{2}+\varepsilon^{2} d_{4} \ddot{\phi} \phi+\varepsilon^{2} d_{5} \dot{\psi}^{2}+\varepsilon^{2} d_{5} \ddot{\psi} \psi-\varepsilon d_{6} \ddot{\Omega}_{1} \\
& -\varepsilon d_{7} \ddot{\Omega}_{2}+\varepsilon^{2} d_{8}\left(\dot{\Omega}_{1}+\dot{\Omega}_{2}\right)^{2}-\varepsilon^{2} d_{9} \dot{\Omega}_{1}^{2}+o\left(\varepsilon^{2}\right) \\
\varepsilon \ddot{\psi}+\varepsilon \eta_{22} \dot{\psi}+\varepsilon\left(1-\varepsilon d_{10} \ddot{\Omega}_{1}-\varepsilon d_{11} \ddot{\Omega}_{2}\right) \psi= & \varepsilon^{2} d_{12} \psi \ddot{\phi}+o\left(\varepsilon^{2}\right)
\end{aligned}
$$

In order to perturb off from the undamped linear equations, the damping terms are scaled by

$$
\eta_{11}=\varepsilon \zeta_{11}, \quad \eta_{22}=\varepsilon \zeta_{22}
$$

so that the scaled equations of motion take the final form as follows

$$
\begin{aligned}
\ddot{\phi}+\omega_{\varphi \beta}^{2} \phi= & -d_{6} \ddot{\Omega}_{1}-d_{7} \ddot{\Omega}_{2}+\varepsilon\left[-\zeta_{11} \dot{\phi}+\left(d_{1} \ddot{\Omega}_{1}+d_{2} \ddot{\Omega}_{2}\right) \phi+d_{3} \dot{\phi}^{2}+d_{4} \ddot{\phi} \phi+d_{5} \dot{\psi}^{2}\right. \\
& \left.+d_{5} \ddot{\psi} \psi+d_{8}\left(\dot{\Omega}_{1}+\dot{\Omega}_{2}\right)^{2}-d_{9} \dot{\Omega}_{1}^{2}\right]+o(\varepsilon) \\
\ddot{\psi}+\psi= & \varepsilon\left[-\zeta_{22} \dot{\psi}+\left(d_{10} \ddot{\Omega}_{1}+d_{11} \ddot{\Omega}_{2}\right) \psi+d_{12} \psi \ddot{\phi}\right]+o(\varepsilon)
\end{aligned}
$$

\section{Nonlinear solution}

\subsection{Perturbation equation}

Since Eqs (34) and (35) do not have a closed form solution, an approximate analytical solution can be generated through perturbation analysis. Using the method of multiple scales, the time scales are defined as

$$
T_{i}=\varepsilon^{i} t^{*} \quad(i=0,1)
$$

A first order approximation to the solutions of Eqs (34) and (35) can be determined in the form

$$
\begin{aligned}
& \phi\left(t^{*}, \varepsilon\right)=\phi_{0}\left(T_{0}, T_{1}\right)+\varepsilon \phi_{1}\left(T_{0}, T_{1}\right) \\
& \psi\left(t^{*}, \varepsilon\right)=\psi_{0}\left(T_{0}, T_{1}\right)+\varepsilon \psi_{1}\left(T_{0}, T_{1}\right)
\end{aligned}
$$

The independent variable $\tau$ is expressed in terms of $T_{0}$ and $T_{1}$ via the chain rule, i.e.

$$
\begin{aligned}
\frac{d}{d t^{*}} & =D_{0}+\varepsilon D_{1} \\
\frac{d^{2}}{d t^{* 2}} & =D_{0}^{2}+2 \varepsilon D_{0} D_{1}
\end{aligned}
$$

where $D_{i}=\partial / \partial T_{i},(i=0,1)$.

Substituting Eqs (37)-(40) into Eqs (34) and (35), then equating coefficients of the same order of $\varepsilon$ in both sides, the following set of differential equations can be obtained.

$\operatorname{Order}\left(\varepsilon^{0}\right)$ :

$$
\begin{aligned}
D_{0}^{2} \phi_{0}+\omega_{\varphi \beta}^{2} \phi_{0} & =-d_{6} D_{0}^{2} \Omega_{1}-d_{7} D_{0}^{2} \Omega_{2} \\
D_{0}^{2} \psi_{0}+\psi_{0} & =0
\end{aligned}
$$


$\operatorname{Order}\left(\varepsilon^{1}\right)$ :

$$
\begin{gathered}
D_{0}^{2} \phi_{1}+\omega_{\varphi \beta}^{2} \phi_{1}=-2 D_{0} D_{1} \phi_{0}-\zeta_{11} D_{0} \phi_{0}+\left(d_{1} D_{0}^{2} \Omega_{1}+d_{2} D_{0}^{2} \Omega_{2}\right) \phi_{0}+d_{3}\left(D_{0} \phi_{0}\right)^{2}+d_{4} D_{0}^{2} \phi_{0} \phi_{0} \\
+d_{5}\left(D_{0} \psi_{0}\right)^{2}+d_{5} D_{0}^{2} \psi_{0} \psi_{0}+d_{8}\left(D_{0} \Omega_{1}+D_{0} \Omega_{2}\right)^{2}-d_{9}\left(D_{0} \Omega_{1}\right)^{2} \\
D_{0}^{2} \psi_{1}+\psi_{1}=-2 D_{0} D_{1} \psi_{0}-\zeta_{22} D_{0} \psi_{0}+\left(d_{10} D_{0}^{2} \Omega_{1}+d_{11} D_{0}^{2} \Omega_{2}\right) \psi_{0}+d_{12} \psi_{0} D_{0}^{2} \phi_{0}
\end{gathered}
$$

Equations (41)-(44) vary with the joint motion and thus are time-varying, which is one of the main differences between the flexible mechanism and the flexible structure.

\subsection{Perturbation solution}

The linear problem is governed by Eqs (41) and (42). Their solutions can be written as

$$
\begin{aligned}
& \phi_{0}=A_{1}\left(T_{1}\right) \exp \left(j \omega_{\varphi \beta} T_{0}\right)-g_{1}+c c \\
& \psi_{0}=A_{2}\left(T_{1}\right) \exp \left(j T_{0}\right)+c c
\end{aligned}
$$

where $A_{1}\left(T_{1}\right)$ and $A_{2}\left(T_{1}\right)$ are functions of slow time $T_{1} ; g_{1}=\left(d_{6} D_{0}^{2} \Omega_{1}+d_{7} D_{0}^{2} \Omega_{2}\right) /\left(2 \omega_{\varphi \beta}^{2}\right) ; c c$ denotes the complex conjugate of the preceding term. Due to smooth rigid motion of these two joints in the flexible manipulator, the third and above derivatives of $\Omega_{1}$ and $\Omega_{2}$ with respect to time are ignored.

The nonlinear problem is governed by Eqs (43) and (44). In order to solve the nonlinear problem, substituting Eqs (45) and (46) into Eqs (43) and (44), the following equations can be obtained.

$$
\begin{aligned}
D_{0}^{2} \phi_{1}+\omega_{\varphi \beta}^{2} \phi_{1}= & -2 j \omega_{\varphi \beta} A_{1}^{\prime} \exp \left(j \omega_{\varphi \beta} T_{0}\right)-\zeta_{11} j \omega_{\varphi \beta} A_{1} \exp \left(j \omega_{\varphi \beta} T_{0}\right)+g_{2}\left[A_{1} \exp \left(j \omega_{\varphi \beta} T_{0}\right)-g_{1}\right] \\
& +d_{3}\left[-A_{1}^{2} \omega_{\varphi \beta}^{2} \exp \left(2 j \omega_{\varphi \beta} T_{0}\right)+A_{1} \bar{A}_{1} \omega_{\varphi \beta}^{2}\right]+d_{4}\left[g_{1} \omega_{\varphi \beta}^{2} A_{1} \exp \left(j \omega_{\varphi \beta} T_{0}\right)\right. \\
& \left.-A_{1}^{2} \omega_{\varphi \beta}^{2} \exp \left(2 j \omega_{\varphi \beta} T_{0}\right)-A_{1} \bar{A}_{1} \omega_{\varphi \beta}^{2}\right]+d_{5}\left[-A_{2}^{2} \exp \left(2 j T_{0}\right)+A_{2} \bar{A}_{2}\right] \\
& +d_{5}\left[-A_{2}^{2} \exp \left(2 j T_{0}\right)-A_{2} \bar{A}_{2}\right]+d_{8}\left(D_{0} \Omega_{1}+D_{0} \Omega_{2}\right)^{2}-d_{9}\left(D_{0} \Omega_{1}\right)^{2}+c c \\
D_{0}^{2} \psi_{1}+\psi_{1}=- & 2 j A_{2}^{\prime} \exp \left(j T_{0}\right)-\zeta_{22} j A_{2} \exp \left(j T_{0}\right)+g_{3} A_{2} \exp \left(j T_{0}\right) \\
+ & d_{12}\left\{-\omega_{\varphi \beta}^{2} A_{1} \bar{A}_{2} \exp \left[j\left(\omega_{\varphi \beta}-1\right) T_{0}\right]-\omega_{\varphi \beta}^{2} A_{1} A_{2} \exp \left[j\left(\omega_{\varphi \beta}+1\right) T_{0}\right]\right\}+c c
\end{aligned}
$$

where, $g_{2}=d_{1} D_{0}^{2} \Omega_{1}+d_{2} D_{0}^{2} \Omega_{2} ; g_{3}=d_{10} D_{0}^{2} \Omega_{1}+d_{11} D_{0}^{2} \Omega_{2}$; (') represents derivatives with respect to the slow time $T_{1}$.

To obtain solutions of Eqs (47) and (48), it is necessary to determine the solvability conditions.

\section{Analysis of internal resonance}

In this section, we will study the responses of the system at the $2: 1$ internal resonance condition, i.e. $\omega_{1} \approx 2 \omega_{2}$. It is this internal resonance condition that enables the transfer of energy between vibration modes. The analysis of the modal amplitudes will verify that the transfer of energy is indeed existent. It will be studied for two cases: undamped and damped.

\subsection{Resonant case, without damping}

In the case of the 2:1 internal resonance, a detuning parameter $\sigma$ is introduced as follow

$$
\omega_{\varphi \beta}=2-\varepsilon \sigma
$$

$2 T_{0}$ and $\left(\omega_{\varphi \beta}-1\right) T_{0}$ are expressed as

$$
\begin{aligned}
2 T_{0} & =\omega_{\varphi \beta} T_{0}+\sigma T_{1} \\
\left(\omega_{\varphi \beta}-1\right) T_{0} & =T_{0}-\sigma T_{1}
\end{aligned}
$$


From Eqs (47) and (48), the solvability conditions are obtained

$$
\begin{array}{r}
-2 j \omega_{\varphi \beta} A_{1}^{\prime}-j \zeta_{11} \omega_{\varphi \beta} A_{1}+\left(g_{2}+d_{4} g_{1} \omega_{\varphi \beta}^{2}\right) A_{1}-2 d_{5} A_{2}^{2} \exp \left(j \sigma T_{1}\right)=0 \\
-2 j A_{2}^{\prime}-\zeta_{22} j A_{2}+g_{3} A_{2}-d_{12} \omega_{\varphi \beta}^{2} A_{1} \bar{A}_{2} \exp \left(-j \sigma T_{1}\right)=0
\end{array}
$$

It is convenient to express the resulting modulation equations in polar form by introducing the following transformation

$$
\begin{aligned}
A_{1} & =\frac{1}{2} a_{1} \exp \left(j \theta_{1}\right) \\
A_{2} & =\frac{1}{2} a_{2} \exp \left(j \theta_{2}\right)
\end{aligned}
$$

where $a_{1}, a_{2}, \theta_{1}, \theta_{2}$ are real functions of the slow time $T_{1} ; a_{1}$ and $a_{2}$ are defined as the modal amplitudes.

Inserting Eqs (54) and (55) into Eqs (52) and (53), then separating the real and imaginary parts, the amplitude and phase modulation equations are obtained

$$
\begin{aligned}
a_{1}^{\prime} & =-\frac{1}{2} \zeta_{11} a_{1}+\frac{1}{2 \omega_{\varphi \beta}} d_{5} a_{2}^{2} \sin \gamma \\
a_{2}^{\prime} & =-\frac{1}{2} \zeta_{22} a_{2}-\frac{1}{4} d_{12} \omega_{\varphi \beta}^{2} a_{1} a_{2} \sin \gamma \\
a_{1} \theta_{1}^{\prime} & =-\frac{a_{1}}{2 \omega_{\varphi \beta}}\left(g_{2}+d_{4} g_{1} \omega_{\varphi \beta}^{2}\right)+\frac{1}{2 \omega_{\varphi \beta}} d_{5} a_{2}^{2} \cos \gamma \\
\theta_{2}^{\prime} & =-\frac{1}{2} g_{3}+\frac{1}{4} d_{12} \omega_{\varphi \beta}^{2} a_{1} \cos \gamma \\
\gamma & =\theta_{1}-2 \theta_{2}-\sigma T_{1}
\end{aligned}
$$

Eliminating $\theta_{1}$ and $\theta_{2}$ from Eqs (56)-(60), yields

$$
a_{1} \gamma^{\prime}=-\frac{a_{1}}{2 \omega_{\varphi \beta}}\left(g_{2}+d_{4} g_{1} \omega_{\varphi \beta}^{2}\right)+\frac{1}{2 \omega_{\varphi \beta}} d_{5} a_{2}^{2} \cos \gamma+g_{3} a_{1}-\frac{1}{2} d_{12} \omega_{\varphi \beta}^{2} a_{1}^{2} \cos \gamma-\sigma a_{1}
$$

In the absence of damping (i.e. $\zeta_{11}=\zeta_{22}=0$ ), we have

$$
\begin{aligned}
& a_{1}^{\prime}=\frac{1}{2 \omega_{\varphi \beta}} d_{5} a_{2}^{2} \sin \gamma \\
& a_{2}^{\prime}=-\frac{1}{4} d_{12} \omega_{\varphi \beta}^{2} a_{1} a_{2} \sin \gamma
\end{aligned}
$$

Let

$$
v=\frac{2 d_{5}}{\omega_{\varphi \beta}^{3} d_{12}}=\frac{2 m_{22}}{m_{11} l_{2}^{2} \omega_{\varphi \beta}^{3}}
$$

Then multiplying Eq. (62) by $a_{1}$ and Eq. (63) by $v a_{2}$, and adding them, yields

$$
a_{1} a_{1}^{\prime}+v a_{2} a_{2}^{\prime}=0
$$

Integrating Eq. (65), we obtain

$$
a_{1}^{2}+v a_{2}^{2}=E=\text { const. }
$$

where $E$ is a integration constant proportional to the initial energy of the system.

Since $v>0$ in Eq. (64), $a_{1}$ and $a_{2}$ in Eq. (66) are always bounded. Because the damping is neglected in this case, the system is conservative and the energy level remains constant. As a result, if the response of the modal amplitude $a_{1}$ is governed by a periodic function, then the modal amplitude $a_{2}$ will be periodic and out of phase with $a_{1}$. Equation (66) demonstrates that, in the absence of damping, the energy in the system continues to be exchanged undamped between the fundamental mode of the flexible link and the vibration mode of the absorber. This conclusion will be further verified by the numerical simulations. 
Table 1

Parameters of the two-link flexible robotic arm

\begin{tabular}{lll}
\hline & Material & Parameters \\
\hline Link1 & Steel & $l_{1}=1.0 \mathrm{~m}$; Square cross-section with side length of $0.05 \mathrm{~m}$. \\
Link2 & Aluminum & $l_{2}=1.0 \mathrm{~m}$; Rectangle cross-section, $h \times b=0.05 \mathrm{~m} \times 0.03 \mathrm{~m} ;$ \\
& & Elastic modulus is $71 \mathrm{GPa}$; Mass density is $2710 \mathrm{~kg} / \mathrm{m}^{3}$. \\
Tip mass & Steel & $m_{B}=0.3 \mathrm{~kg}$. \\
\hline
\end{tabular}

\subsection{Resonant case, with damping}

In the presence of damping (i.e. $\zeta_{11}>0$ and $\zeta_{22}>0$ ), the equilibrium points for this case are defined by the solution of the set of steady state secular term equations, namely

$$
\begin{aligned}
& -\frac{1}{2} \zeta_{11} a_{1}+\frac{1}{2 \omega_{\varphi \beta}} d_{5} a_{2}^{2} \sin \gamma=0 \\
& -\frac{1}{2} \zeta_{22} a_{2}-\frac{1}{4} d_{12} \omega_{\varphi \beta}^{2} a_{1} a_{2} \sin \gamma=0 \\
& -\frac{a_{1}}{2 \omega_{\varphi \beta}}\left(g_{2}+d_{4} g_{1} \omega_{\varphi \beta}^{2}\right)+\frac{1}{2 \omega_{\varphi \beta}} d_{5} a_{2}^{2} \cos \gamma+g_{3} a_{1}-\frac{1}{2} d_{12} \omega_{\varphi \beta}^{2} a_{1}^{2} \cos \gamma-\sigma a_{1}=0
\end{aligned}
$$

By inspection, it is determined that the system posses an infinite number of equilibrium points defined by

$$
a_{1}=0, \quad a_{2}=0, \quad \gamma \in R
$$

Therefore, by evaluating the Jacobian, we can ascertain the stability of the system.

The Jacobian matrix for this case is

$$
\left[\begin{array}{ccc}
\mu_{1} & 0 & 0 \\
0 & \mu_{2} & 0 \\
0 & 0 & 0
\end{array}\right]
$$

where $\mu_{1}=-\frac{1}{2} \zeta_{11}, \mu_{1}=-\frac{1}{2} \zeta_{22}$.

The corresponding eigenvalues are $\left(\mu_{1}, \mu_{2}, 0\right)$. Obviously, $\mu_{1}<0$ and $\mu_{2}<0$. Therefore, the modal amplitudes $a_{1}$ and $a_{2}$ are stable, as indicated by the negative eigenvalues.

By numerical integrations of Eqs (56)-(60), it is shown that, in the presence of damping, the energy in the system continues to be exchanged between the fundamental mode of the flexible link and the vibration mode of the absorber, but it is continuously dissipated. Therefore, modal interaction can be used as a feasible way to control vibration of the flexible arm. This conclusion will be further verified by the numerical simulations.

\section{Simulation and analysis}

To verify the above theoretical analysis, a two-link robotic arm with last link flexible is used in the following numerical simulations, as shown in Fig. 1. Link1 is a uniform rigid beam and Link2 is a uniform Euler-Bernoulli flexible beam. Their parameters are listed in Table1. Only the in-plane flexural deformation $\delta\left(x_{2}, t\right)$ about $y_{2}$ axis in the plane $\mathrm{O}_{2} x_{2} y_{2}$ is considered.

Suppose the desired joint motion of the manipulator is

$$
\left\{\begin{array}{l}
q_{1}=0.1 \sin (\pi t / 7) \\
q_{2}=0.1 \cos (\sqrt{2} t / 5)
\end{array},(0 \leqslant t \leqslant 100 \mathrm{~s})\right.
$$

At $t=10 \mathrm{~s}$, a moving object $M_{0}$ with the mass of $0.05 \mathrm{~kg}$ impacts the endpoint of Link2 in the plane $o_{2} x_{2} y_{2}$, perpendicular to $x_{2}$ axis, as shown in Fig. 4. Its relative velocity with respect to the end-effector is $0.3 \mathrm{~m} / \mathrm{s}$ and impact time period is $0.05 \mathrm{~s}$. The corresponding vibration of the endpoint is shown in Fig. 5. It is seen that impact 


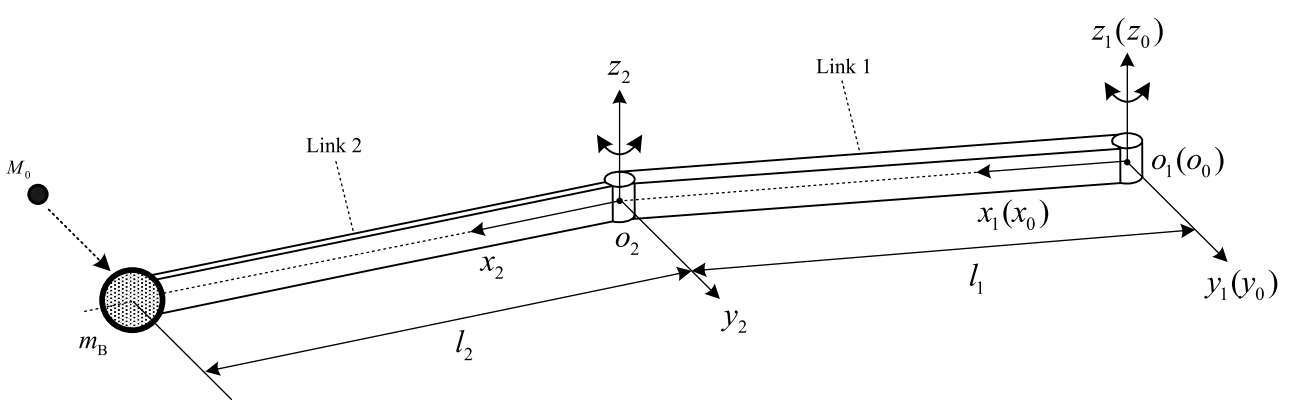

Fig. 4. Schematic of the flexible manipulator without vibration absorber.

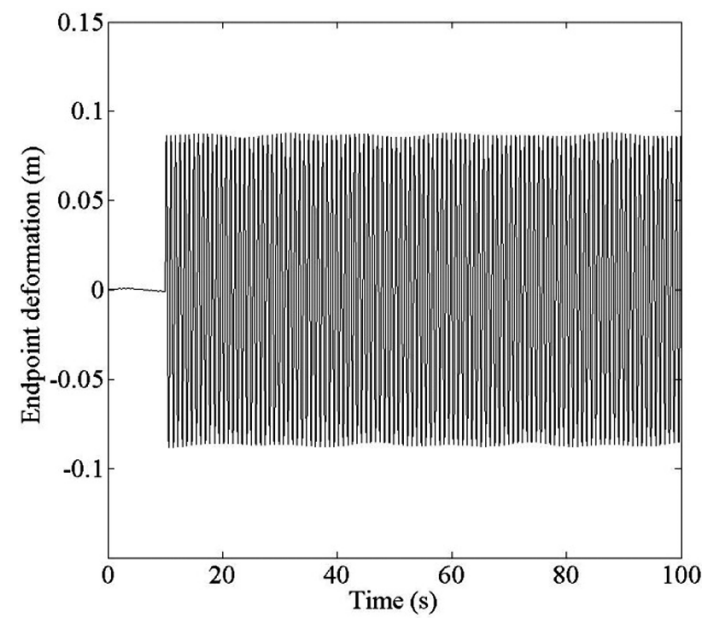

Fig. 5. Endpoint vibration undergoing impact.

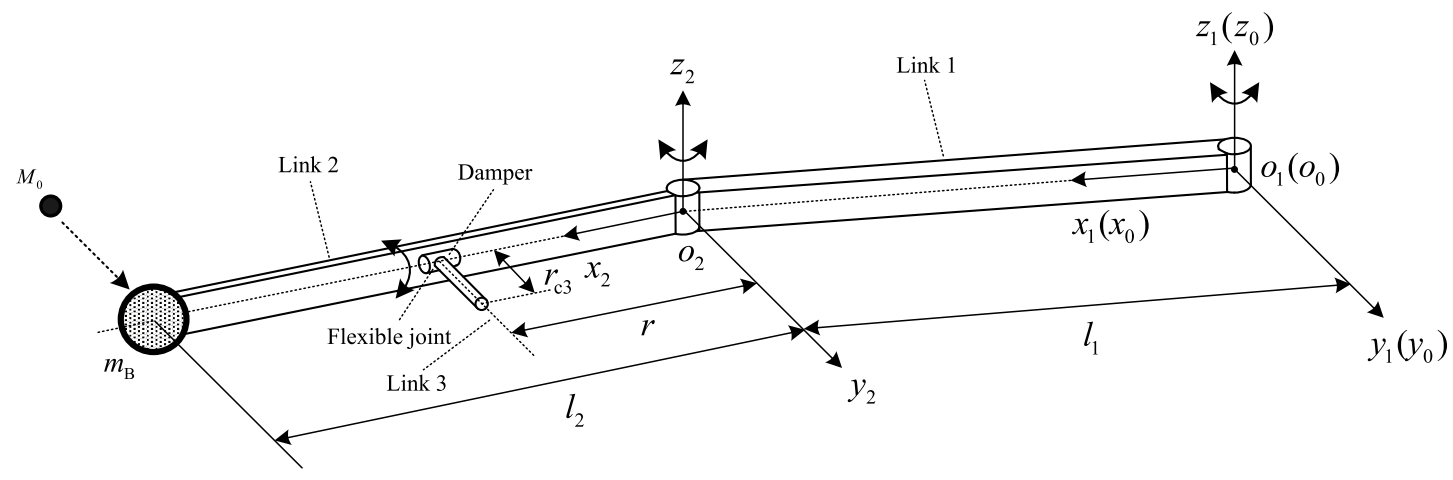

Fig. 6. Schematic of the flexible manipulator with vibration absorber.

vibration responses arise suddenly due to abrupt changes of system velocities in the impact phase. Furthermore, since the manipulator is required to keep moving after the collision, new vibration responses inevitably arise and are accumulated on the existing impact vibration responses. In this example, the largest vibration deformation of the endpoint is $0.0881 \mathrm{~m}$. Due to low structural damping, vibration responses cannot be decreased.

In order to reduce impact vibration via internal resonance, a vibration absorber is attached to Link2 at $r=0.5 \mathrm{~m}$, which mass $m_{3}=0.1 \mathrm{~kg}$ and centroid position $r_{c 3}=0.2 \mathrm{~m}$, as shown in Fig. 6 . The same impact parameters as the aforementioned case are used. At the state of 2:1 internal resonance, Eqs (56)-(60) are integrated numerically in the absence of damping. The relationship between the modal amplitude $a_{1}$ (solid line) and $a_{2}$ (dashed line) is shown in 


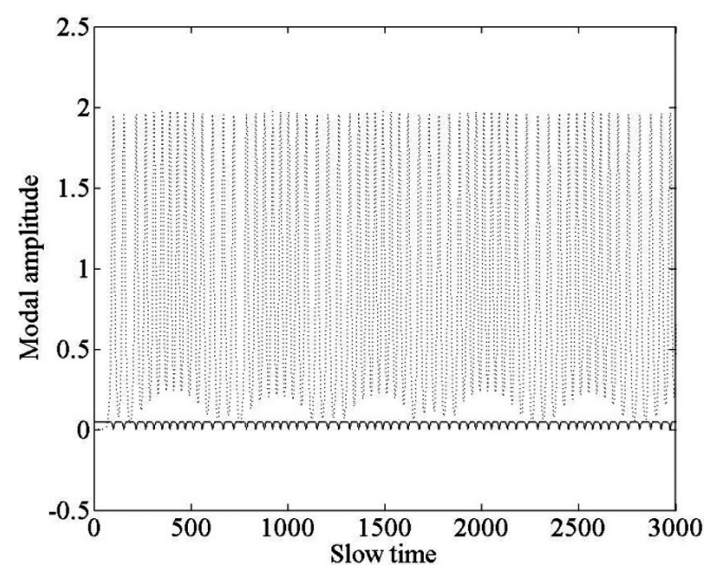

Fig. 7. Undamped modal amplitudes.

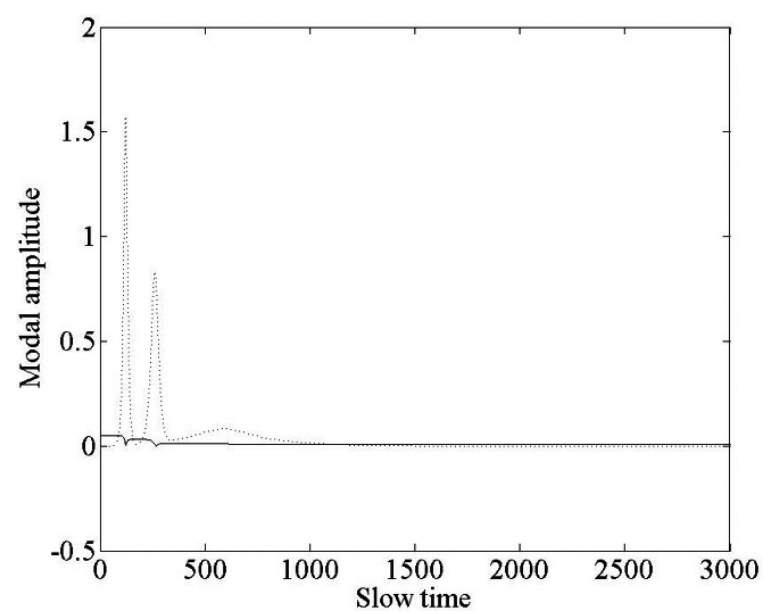

Fig. 8. Damped modal amplitudes.

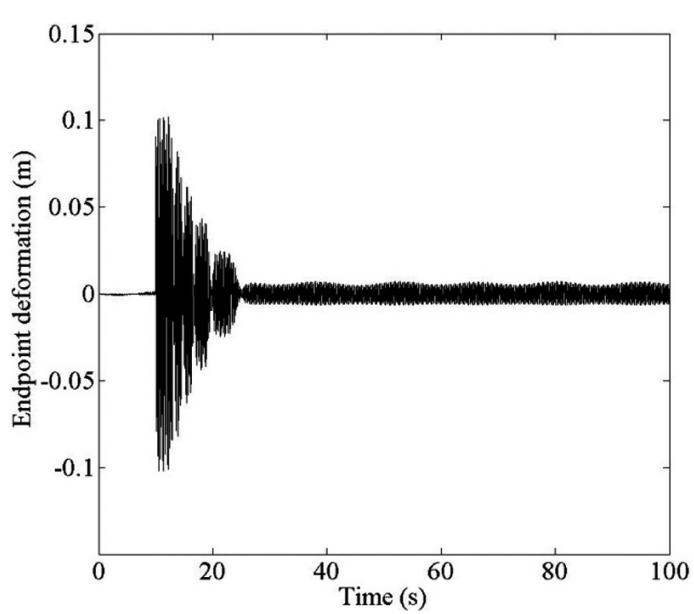

Fig. 9. Endpoint response of the controlled arm.

Fig. 7. It can be seen that the peaks and troughs of the responses are exactly $180^{\circ}$ out of phase, which means that there is continuous exchange of the energy between vibration modes.

When the damping of the vibration absorber is taken into account, letting $c_{2}=0.001$, Eqs (56) $-(60)$ are integrated numerically at the state of 2:1 internal resonance. The modal amplitude $a_{1}$ (solid line) and $a_{2}$ (dashed line) are illustrated in Fig. 8. It is shown that a transfer of energy between modes is occurring, verified by the coincidence of the peaks of one modal curve with the troughs of the other. In addition, the modal amplitudes decay with time and approach the equilibrium value $\left(a_{1}=a_{2}=0\right)$, demonstrating that the vibrational energy of the flexible arm has been effectively absorbed and dissipated through modal interaction.

The endpoint deformation of the flexible arm equipped with the vibration absorber is shown in Fig. 9. At the state of 2:1 internal resonance, the endpoint deformation is decreased quickly by $50 \%$ within 7 seconds, $75 \%$ within 10 seconds, $90 \%$ within 15 seconds, compared with the uncontrolled case (as shown in Fig. 5). It is shown that this control method can effectively decrease large amplitude impact vibration of the flexible manipulator.

Moreover, when a vibration absorber is incorporated into a flexible manipulator, several parameters are also introduced accordingly, including: mass of the rigid link $\left(m_{3}\right)$, damping of the vibration absorber $\left(c_{2}\right)$, centroid position of the rigid link $\left(r_{c 3}\right)$ (i.e. the distance from the centroid to the joint of the rigid link), joint position of the rigid link $(r)$ (i.e. the distance from the joint of the rigid link to the joint of the hinged flexible link), as shown in Fig. 3(a). These parameters play important roles in improving dynamic performance of the flexible manipulator. As a result, it is necessary to seek appropriate parameter values to achieve optimal control effect. In this study, the aim 


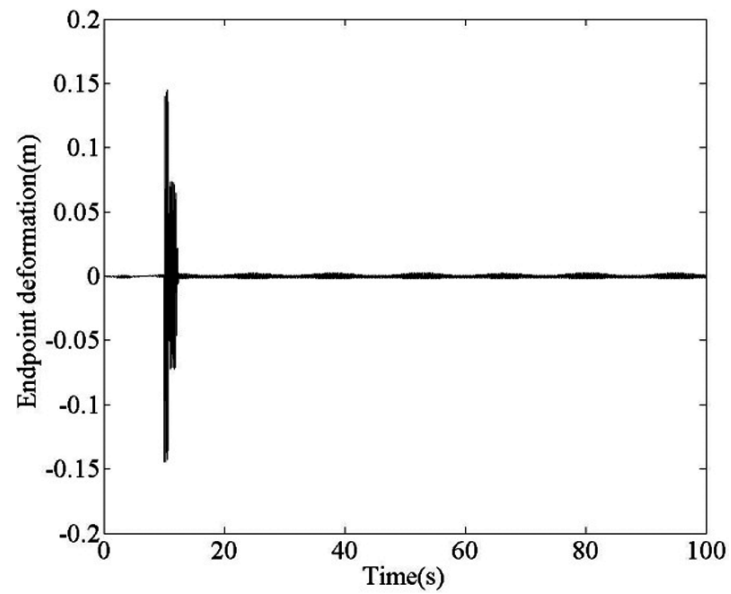

Fig. 10. Endpoint response of the controlled arm.

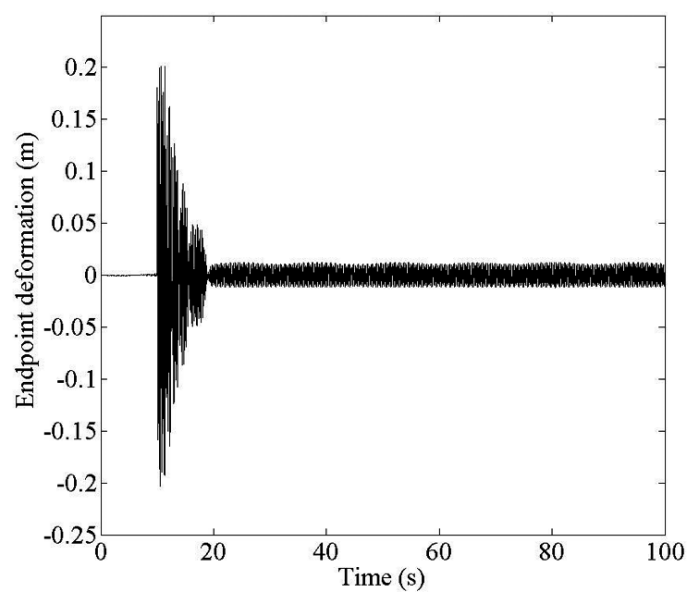

Fig. 11. Endpoint response of the controlled arm.

of this control method is to attenuate the impact vibration quickly and effectively. Therefore, the optimization object and constraints are

$$
\begin{aligned}
& \min \rightarrow t_{a} \\
& \text { s.t. } m_{3 \_ \text {min }} \leqslant m_{3} \leqslant m_{3 \_ \text {max }} \\
& c_{2 \_ \text {min }} \leqslant c_{2} \leqslant c_{2 \_ \text {max }} \\
& r_{c 3 \_ \text {min }} \leqslant r_{c 3} \leqslant r_{c 3 \_\max } \\
& r_{\text {min }} \leqslant r \leqslant r_{\max }
\end{aligned}
$$

where $t_{a}$ is the attenuation time when the impact vibration response is decreased to a certain threshold (e. g. $0.01 \mathrm{~m}$ in this simulation), obviously small $t_{a}$ means quick suppression; $m_{3_{-} \text {min }}$ and $m_{3_{-} \text {max }}$ are the lower and upper permitted mass of the rigid link; $c_{2}$ min and $c_{2}$ max are the lower and upper permitted damping of the vibration absorber; $r_{c 3_{-} \min }$ and $r_{c 3_{-} \max }$ are the lower and upper permitted centroid position of the rigid link; $r_{\min }$ and $r_{\max }$ are the lower and upper permitted joint position of the rigid link.

This is a multi-variable optimization problem and an optimization algorithm based on Particle swarm optimization (PSO) is used to obtain the optimal parameters. Several important parameter values are chosen as follows: swarm size is 20; particle dimension is 4, i.e. $m_{3}, c_{2}, r_{c 3}$ and $r$; cognitive acceleration and social acceleration are 2 respectively; value of inertial weight at the beginning is 0.95 ; value of inertial weight at the end of the PSO iterations is $0.4 ; 0.05 \mathrm{~kg} \leqslant m_{3} \leqslant 0.3 \mathrm{~kg}, 0.0001 \leqslant c_{2} \leqslant 0.001,0.1 \mathrm{~m} \leqslant r_{c 3} \leqslant 0.5 \mathrm{~m}, 0.2 \mathrm{~m} \leqslant r \leqslant 0.8 \mathrm{~m}$. The same impact parameters as the aforementioned case are used and the corresponding optimization results are obtained, i.e. $m_{3}=0.0507 \mathrm{~kg}, c_{2}=0.0009, r_{c 3}=0.1031 \mathrm{~m}, r=0.6013 \mathrm{~m}$. In this case, the endpoint deformation is calculated and shown in Fig. 10, in which the vibration deformation is decreased to $0.01 \mathrm{~m}$ within 2.3 second and the final residual deformation is $0.005 \mathrm{~m}$. Compared with the case without optimization (as shown in Fig. 9), in which the vibration deformation is decreased to $0.01 \mathrm{~m}$ within 15 second, the attenuation time is decrease by about $85 \%$ and the final residual deformation is decreased by about $50 \%$. It is demonstrated that this control method can attenuate large amplitude impact vibration of the flexible manipulator more quickly and effectively with optimality parameters.

In addition, an example of the relative impact velocity $0.6 \mathrm{~m} / \mathrm{s}$ is conducted. The endpoint deformation is shown in Fig. 11. As can be seen, even though larger impact velocity is imposed, vibration response can still be quelled effectively and quickly.

Through above simulation and analysis, it is verified that this control method based on internal resonance is effective in quelling large amplitude impact vibration of the flexible manipulator undergoing rigid motion. 


\section{Conclusion}

Due to low flexibility, impact can excite severe large amplitude vibration responses of the flexible robotic manipulator. Since this impact vibration exhibits characteristics of remarkable nonlinearity and strong energy, it is necessary to seek an effective vibration absorption method based on appropriate nonlinear principle.

This study aims to put forward an energy-based control method to absorb and attenuate large amplitude impact vibration of the flexible manipulator. The method takes advantage of internal resonance and is implemented through a vibration absorber based on the transfer and dissipation of energy. The addition of a vibration absorber to the flexible arm introduces quadratic nonlinearties into the system, thereby generating a coupling effect between vibration modes of the system. It is verified that internal resonance can be established for the flexible manipulator undergoing rigid motion. Its theoretical feasibility of controlling large amplitude impact vibration of the flexible manipulator is explored and its stability is analyzed. By means of analysis on 2:1 internal resonance, the exchange of energy is proven to be existent. The impact vibrational energy can be transferred from the arm to the absorber and dissipated through the damping of the absorber.

The results of numerical simulations are promising and preliminarily verify that the method is feasible and can be used to combat large amplitude impact vibration of the flexible manipulator subject to rigid motion.

\section{Acknowledgments}

This study is supported by National Natural Science Foundation of China (Grant No.51105015, 51075013), Beijing Natural Science Foundation (Grant No.4102035), National Key Technology R\&D Program of China (Grant No.2011BAF04B00).

\section{References}

[1] S.K. Ider, M.K. Özgören and V. Ay, Trajectory tracking control of robots with flexible links, Mechanism and Machine Theory 37(11) (2002), 1377-1394.

[2] S. Kilicaslan, M.K. Özgören and S.K. Ider, Hybrid force and motion control of robots with flexible links, Mechanism and Machine Theory 45(1) (2010), 91-105.

[3] S.K. Ider and O. Korkmaz, Trajectory tracking control of parallel robots in the presence of joint drive flexibility, Journal of Sound and Vibration 319(1-2) (2008), 77-90.

[4] G. Gilardi and I. Sharf, Literature survey of contact dynamics modeling, Mechanism and Machine Theory 37(10) (2002), 1213-1239.

[5] J. Kovecses and W.L. Cleghorn, Impulsive dynamics of a flexible arm: analytical and numerical solutions, Journal of Sound and Vibration 269(1-2) (2004), 183-195.

[6] S.A.M. Najafabadi, J. Kovecses and J. Angeles, Impacts in multibody systems: Modeling and experiments, Multibody System Dynamics 20(2) (2008), 163-176.

[7] K.D. Bhalerao and K.S. Anderson, Modeling intermittent contact for flexible multibody systems, Nonlinear Dynamics 60(1-2) (2010), 63-79.

[8] K. Dupree, G. Hu and W.E. Dixon, Adaptive Lyapunov-based control of a robot and mass-spring system undergoing an impact collision, IEEE Transactions on Systems, Man and Cybernetics-Part B: Cybernetics 38(4) (2008), 1050-1061.

[9] P.R. Pagilla and B. Yu, An experimental study of planar impact of a robot manipulator, IEEE/ASME Transactions on Mechatronics 9(1) (2004), 123-128

[10] K. Shimamoto, N. Takeuchi and H. Lim, Development of collision force suppression mechanism for human-friendly robot, 11th International Conference on Control, Automation and Systems (2011), 665-670.

[11] M. Uemura and S. Kawamura, Passivity-based controllers for periodic motions of multi-joint robots with impact phenomena, IEEE/RSJ International Conference on Intelligent Robots and Systems (2010), 664-669.

[12] A.D. Luca and L. Ferrajoli, Exploiting robot redundancy in collision detection and reaction, IEEE/RSJ International Conference on Intelligent Robots and Systems (2008), 3299-3305.

[13] A.D. Luca and R. Mattone, Sensorless robot collision detection and hybrid force/motion control, IEEE International Conference on Robotics and Automation (2005), 999-1004.

[14] K. Ziaei and D.W.L. Wang, Design and experimental evaluation of a single robust position-force controller for a single flexible link (SFL) manipulator in collision, IEEE International Conference on Robotics and Automation 3 (2003), 3114-3119.

[15] A. Garcia and V. Feliu, Force control of a single-link flexible robot based on a collision detection mechanism, IEE Proceedings: Control Theory and Applications 147(6) (2000), 588-595.

[16] A. Garcia, V. Feliu and J.A. Somolinos, Gauge based collision detection mechanism for a new three-degree-of-freedom flexible robot, IEEE International Conference on Robotics and Automation (2001), 3853-3858. 
[17] W.L. Xu and S. Yue, Pre-posed configuration of flexible redundant robot manipulators for impact vibration alleviating, IEEE Transactions on Industrial Electronics 51(1) (2004), 195-200.

[18] S. Liu, L. Wu and Z. Lu, Impact dynamics and control of a flexible dual-arm space robot capturing an object, Applied Mathematics and Computation 185(2) (2007), 1149-1159.

[19] M. Benosman and G.L. Vey, Control of flexible manipulators: A survey, Robotica 22(5) (2004), 533-545.

[20] S.K. Dwivedy and P. Eberhard, Dynamic analysis of flexible manipulators, a literature review, Mechanism and Machine Theory 41(7) (2006), 749-777.

[21] A.H. Nayfeh. Nonlinear interactions, New York: Wiley Interscience, 2000.

[22] F. Wang and A.K. Bajaj, Nonlinear dynamics of a three-beam structure with attached mass and three-mode interactions, Nonlinear Dynamics 62(1-2) (2010), 461-484

[23] Y. Du, R.A. Burdisso and E. Nikolaidis, Control of internal resonances in vibration isolators using passive and hybrid dynamic vibration absorbers, Journal of Sound and Vibration 286(4-5) (2005), 697-727.

[24] M.F. Daqaq, E.M. Abdel-Rahman and A.H. Nayfeh, Two-to-one internal resonance in microscanners, Nonlinear Dynamics 57(1-2) (2009), $231-251$.

[25] O. Thomas, C. Touze and E. Luminais, Non-linear vibrations of free-edge thin spherical shells: Experiments on a 1:1:2 internal resonance, Nonlinear Dynamics 49(1-2) (2007), 259-284.

[26] X. Hao and L. Xu, Internal resonance analysis for electromechanical integrated toroidal drive, Journal of computational and Nonlinear Dynamics 5(4) (2010), 1-12.

[27] S.K. Dwivedy and R.C. Kar, Nonlinear dynamics of a cantilever beam carrying an attached mass with 1:3:9 internal resonances, Nonlinear Dynamics 31(1) (2003), 49-72.

[28] M.F. Golnaraghi, Regulation of flexible structures via nonlinear coupling, Dynamics and Control 1(4) (1991), $405-428$.

[29] M.F. Golnaraghi, K.L. Tuer and D. Wang, Regulation of a lumped parameter cantilever beam via internal resonance using nonlinear coupling enhancement, Dynamics and Control 4(1) (1994), 73-96.

[30] K.L. Tuer, A.P. Duquette and M.F. Golnaraghi, Vibration control of a flexible beam using a rotational internal resonance controller, part I: Theoretical development and analysis, Journal of Sound and Vibration 167(1) (1993), 41-62.

[31] A.P. Duquette, K.L. Tuer and M.F. Golnaraghi, Vibration control of a flexible beam using a rotational internal resonance controller, part II: Experiment, Journal of Sound and Vibration 167(1) (1993), 63-75.

[32] K.L. Tuer, M.F. Golnaraghi and D. Wang, Development of a generalised active vibration suppression strategy for a cantilever beam using internal resonance, Nonlinear Dynamics 5(2) (1994), 131-151.

[33] S.S. Oueini, K.L. Tuer and M.F. Golnaraghi, Regulation of a two-degree-of-freedom structure using internal resonance, Journal of Dynamic Systems, Measurement and Control 117(2) (1995), 247-251.

[34] S.S. Oueini and M.F. Golnaraghi, Experimental implementation of the internal resonance control strategy, Journal of Sound and Vibration 191(3) (1996), 377-396.

[35] Y. Bian, Research on dynamics and control of flexible redundant manipulators, Dissertation for Ph.D, Beihang University, Beijing, China, 1998. 

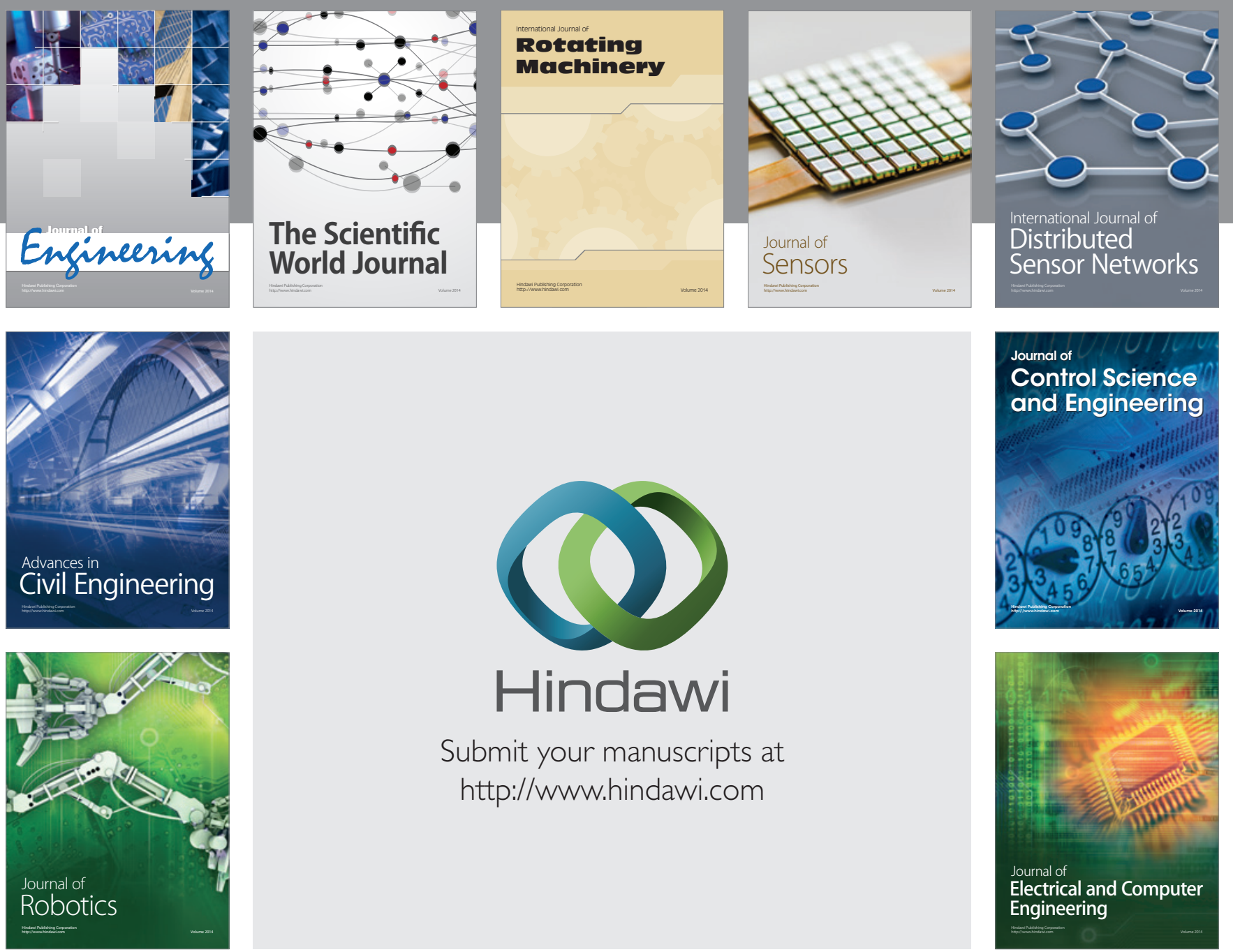

Submit your manuscripts at

http://www.hindawi.com
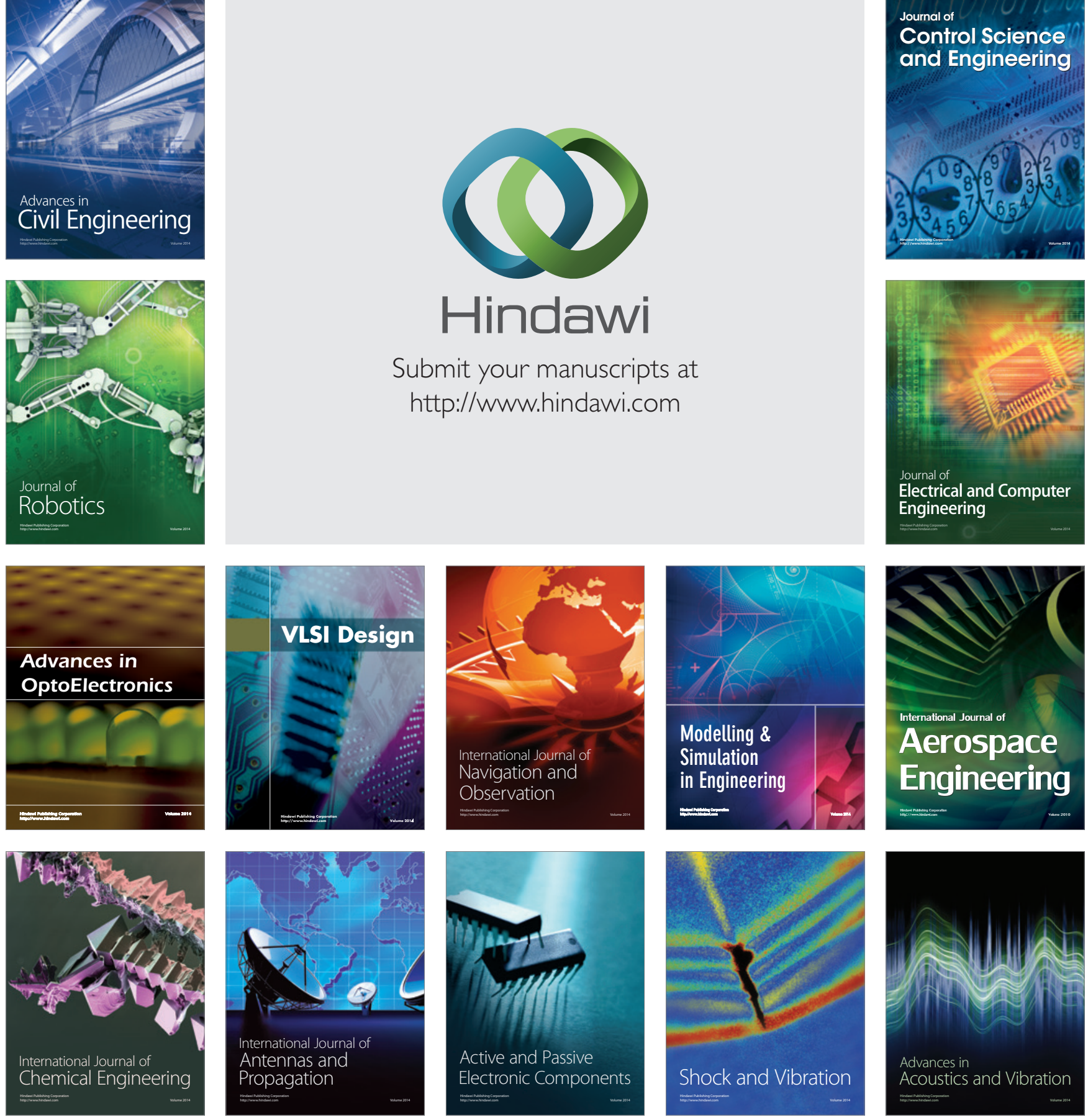